\title{
Nanoscale and Single-Dot Patterning of Colloidal Quantum Dots
}

\author{
Weiqiang Xie, ${ }^{\dagger, \ddagger}$ Raquel Gomes, ${ }^{\ddagger}$, Tangi Aubert, ${ }^{\ddagger}, \S$ Suzanne Bisschop, ${ }^{\ddagger}, \S$ Yunpeng Zhu, ${ }^{\dagger, \ddagger}$ Zeger Hens, \\ Edouard Brainis, ${ }^{\ddagger}, \S$ and Dries Van Thourhout ${ }^{*},+$, \\ ${ }^{\dagger}$ INTEC-department and ${ }^{\ddagger}$ Center for Nano- and Biophotonics (NB-Photonics), Ghent University, Sint-Pietersnieuwstraat 41, Ghent \\ 9000, Belgium \\ ${ }^{\S}$ Physics and Chemistry of Nanostructures, Ghent University, Krijgslaan 281-S3, 9000 Ghent, Belgium
}

\section{Supporting Information}

ABSTRACT: Using an optimized lift-off process we develop a technique for both nanoscale and single-dot patterning of colloidal quantum dot films, demonstrating feature sizes down to $\sim 30 \mathrm{~nm}$ for uniform films and a yield of $40 \%$ for single-dot positioning, which is in good agreement with a newly developed theoretical model. While first of all presenting a unique tool for studying physics of single quantum dots, the process also provides a pathway toward practical quantum dotbased optoelectronic devices.

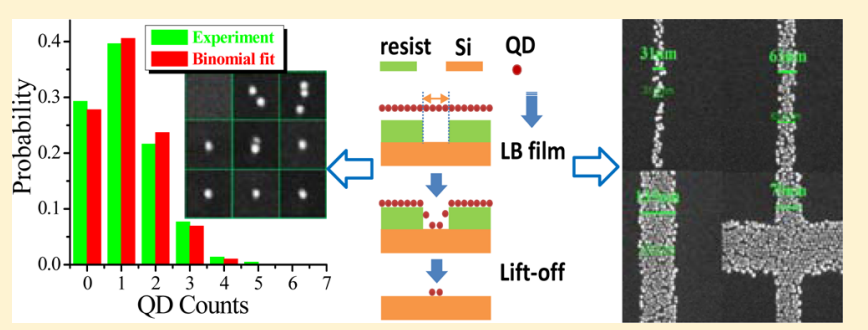

KEYWORDS: Colloidal quantum dots, nanoscale patterning, single-dot positioning, Langmuir-Blodgett, lift-off

olloidal semiconductor nanocrystals or quantum dots (QDs), mostly synthesized by wet chemistry, have emerged as a novel class of materials of high scientific and technological interest. Their nanoscale dimensions give rise to strong quantum confinement effects and concomitantly unique electrical and optical properties. In particular, these colloidal QDs exhibit widely tunable absorption and emission spectra. Combined with their suitability for solution-based processing, which makes them compatible with a wide range of existing material technologies, this makes for an ideal platform for fundamental studies in nanoscience and a wide variety of potential applications such as integrated photonics, ${ }^{1,2}$ lasers, 3,4 light-emitting diodes, ${ }^{5,6}$ photodetectors, ${ }^{7}$ and biosensing. ${ }^{8}$ Whereas colloidal QDs are synthesized as colloidal dispersions, multiple applications need QD films with different requirements in terms of film area, thickness, and patterning. As a result, the geometrically controlled deposition of QD monoand multilayers is an essential step for exploiting their unique properties in practical applications. For instance, the quantitative analysis of the interaction between active QDs and photonic components such as optical waveguides, ${ }^{2}$ resonators, ${ }^{1,9,10}$ and surface plasmons ${ }^{11}$ requires well-controlled pattern shape and accurate placement of QDs integrating with passive photonics. Well-controlled approaches to deposit QDs in nanoscale patterns can also enable QD-based devices such as light-emitting diodes, photodetectors, modulators, or sensors to be further miniaturized and eventually integrated on photonic chips.

Parallel to application development, the investigation of QD properties at the single-dot level has become of significant importance to deepen the understanding of some of their fundamental optoelectronic properties and their interaction with external environments. Seminal studies have addressed the dynamical and spectral properties of single QDs, either through photoexcitation or via tunneling spectroscopy ${ }^{12-20}$ and their coupling to microcavities, ${ }^{21}$ plasmonic nanocavities, ${ }^{22}$ or optical antennas. $^{23-26}$ The QD properties these investigations revealed, such as single photon emission ${ }^{12,13,21}$ and tunable radiation by coupling with photonic building blocks, ${ }^{23-26}$ may eventually promote the development of single-QD based devices, such as single photon sources and controlled quantum emitters. Currently, most of the achieved investigations with single QDs rely either on randomly deposited QDs and later careful localization of target single-QD devices, or on directly patterning QDs close to the predefined structures. Both random approach and existing direct-patterning of QDs, however, have very low yield of localizing single QDs and lack an accurate control over the QD position, thus limiting the experimental efficiency and hampering the development of single-QD devices. Therefore, one of the biggest challenges for single-QD studies, and eventually for single-QD devices, is the ability to position a single $\mathrm{QD}$ at a predefined location with an accuracy of a few tens of nanometers or less.

Various approaches to deposit QDs in micrometer- and nanometer-sized patterns have been proposed in literature, using such methods as optical lithography combined with Langmuir-Blodgett (LB) deposition, ${ }^{27}$ direct electron-beam lithography (EBL) on LB films ${ }^{28}$ or combined with a lift-off process, ${ }^{29-31}$ electrostatic self-assembly techniques, ${ }^{32,33}$ and lithography assisted by surface functionalization. ${ }^{1,25}$ However, despite the significant progress made in patterning QDs, the technique still remains to be improved in terms of the control

Received: August 3, 2015

Revised: October 2, 2015

Published: October 12, 2015 
(a)

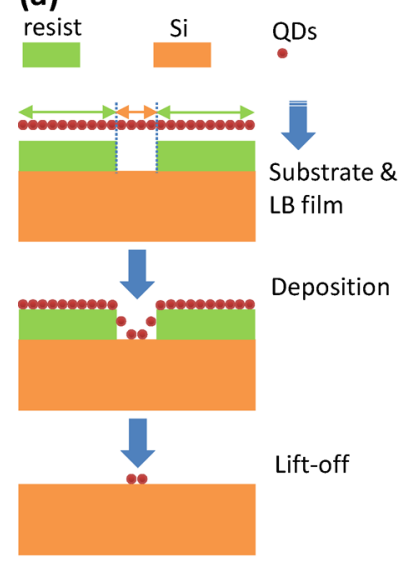

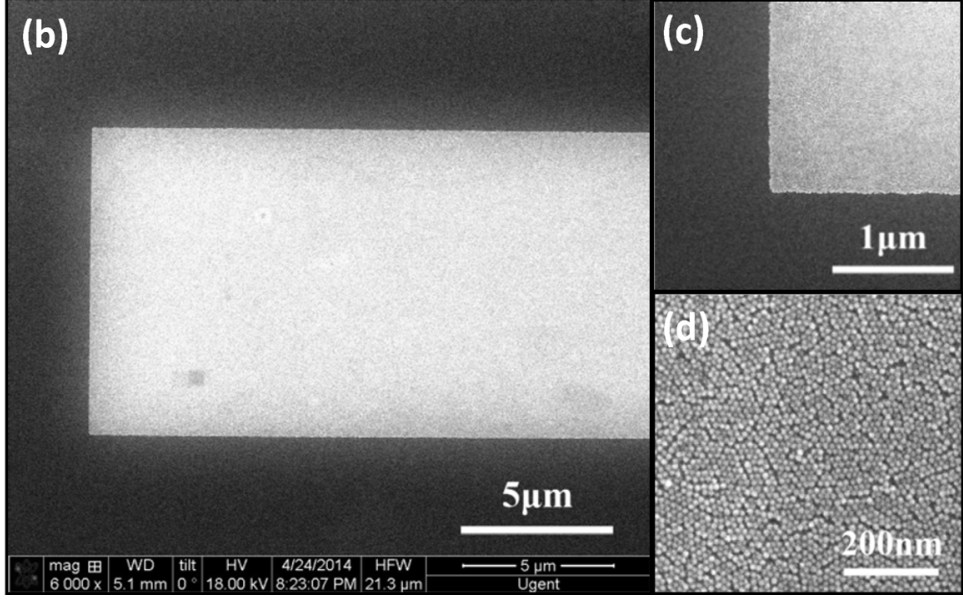

Figure 1. (a) Schematics of the experimental flow of the patterning of QDs. (b-d) SEM images of the microscale patterns of LB QD film after liftoff.

over patterning geometry and dimension, and the quality of the patterned layer. For instance, among most demonstrated experiments for QDs pattering, it remains a challenge to achieve the ultrauniform and contamination-free patterns that applications will eventually need. Moreover, little approaches have demonstrated a straightforward and high yield positioning of individual QD. Often, downscaling a methodology to singleQD positioning leads to significant complications due to the extremely small size of QDs and single-QD deposits typically come together with ill-defined QD patches.

Here, we introduce an EBL-based technique for the formation of nanopatterned QD monolayers providing excellent control over the pattern structure and maintaining a well-defined $\mathrm{QD}$ surface density in the monolayer. By combining photoresist patterning by EBL, LB deposition of QD monolayers and an optimized lift-off process, we demonstrate QD patterns with feature sizes down to $\sim 30 \mathrm{~nm}$ and with arbitrary yet predefined shapes. The patterned QD monolayer exhibits sharp edges and the process leaves the monolayer and the blank substrate free of residues. Moreover, we show that the same approach applies to the formation of single-QD patterns with a single-QD deposition yield as high as $40 \%$. Importantly, the approach relies on methodologies compatible with standard solid-state pre- and postprocessing steps that can be applied on large area substrates. A numerical model describing the statistical behavior of the QD deposition process is developed and shows excellent agreement with the experimental results. The nano- and single- QD patterning technology proposed here not only provides a powerful tool for the fundamental studies of QDs but also represents a significant step toward optoelectronic devices based on QD nanopatterns and, eventually, single QDs.

Figure 1a schematically illustrates the proposed processing scheme. First, using EBL we defined the desired pattern in a diluted ZEP 520A resist film with an initial thickness of $\sim 40$ $\mathrm{nm}$. Any possible residues were removed using a few seconds of oxygen plasma. If a thinner resist film is needed, the oxygen plasma was applied for longer time. Next a close-packed monolayer of QDs was formed through a LB trough (Nima 312D) and then transferred onto the patterned substrate, by pulling the substrate out of the LB trough. We used oleate passivated CdSe/CdS core/shell QDs synthesized by a seededgrowth flash approach ${ }^{34}$ with a diameter of $\sim 10 \mathrm{~nm}$ and a central emission peak of $\sim 650 \mathrm{~nm}$. Finally, a lift-off process was carried out and the resist was removed from the substrate leaving the patterned QDs behind. The quality of the QD pattern was examined by a high-resolution scanning electron microscope (SEM) (FEI Nova 600) and photoluminescence (PL) characterization on a micro-PL setup.

In comparison with spin-coating, the LB deposition of QDs used here provides better control of both the quality and the exact thickness of the QD film. With spin-coating, whereby the QD film is formed directly from the solution by evaporating the solvent, it is impossible to obtain a uniform layer of QDs and the resultant film is typically either submonolayer in nature or consists of areas with multilayers of varying thickness. Spinning QDs on a patterned substrate unavoidably results in over deposition of QDs near steps or a discontinuous film around pattern edges. As a result the subsequent lift-off process is made difficult by the limited amount of dissolvent seeping through the accumulated QD layer near steps or the film might completely peel off from the patterned areas. In contrast, the LB technique allows preparing a highly uniform monolayer of QDs over large areas, even when these are not completely flat, as long as the height variations are sufficiently gradual. When depositing the LB film onto a patterned substrate with sharp edges, the film around the patterned location will bend and break up as schematically indicated in Figure 1a, resulting in exposed sidewalls favorable for the subsequent lift-off process. On the other hand, due to additional area associated with the sidewalls, the QD density within the area of the pattern will be smaller than that of a closed packed film as illustrated in Figure 1a. This effect is illustrated by SEM images of the substrate after QD deposition but before resist lift-off (see Figure S1 in the Supporting Information). It can be seen that the resist is covered by an undisturbed LB film up to the pattern edges whereas part of the QDs within the pattern stick to the resist sidewalls.

Obviously, to minimize this effect the resist thickness needs to be thinned down. However, there is a trade-off between reducing the thickness of the resist and establishing an effective lift-off process without residues: the thicker the resist, the easier and cleaner the lift-off, and vice versa. When the thickness of the resist becomes comparable to the size of the QDs, lifting off QDs from the substrate becomes nearly impossible since all openings are covered by the close-packed QD film and no 

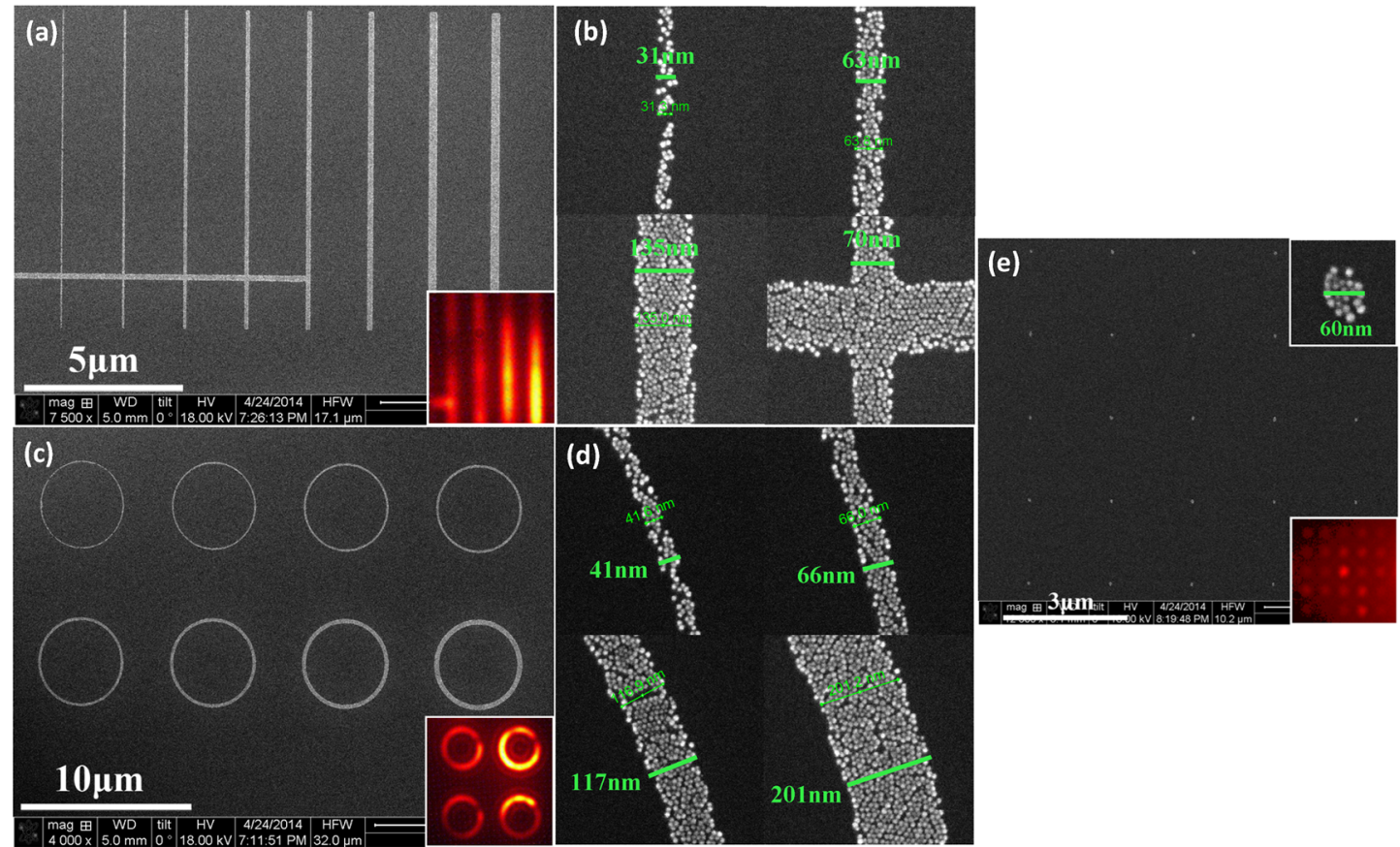

Figure 2. SEM images of the nanoscale patterns for QD films. (a) Line and (c) ring patterns with different widths, and the enlarged views for some selected widths for line (b) and ring (d) patterns, respectively. (e) The array of dot patterns with an average diameter of $60 \mathrm{~nm}$ and the enlarged image of one dot in the inset at right upper corner. The insets at the right lower corner in $(\mathrm{a}, \mathrm{c}, \mathrm{e})$ show the respective micro-PL of the corresponding pattern shapes.

dissolvent can seep into the resist to start of the lifting process. More importantly, because of the strong van der Waals' interaction $^{27}$ between the QDs and the substrate, the lifted QDs can redeposit on the substrate from the dissolvent, an effect much more notable for thinner resists. Therefore, an efficient lift-off procedure turns out to be critical in obtaining a contaminant-free patterning and the associated threshold thickness for the resists needs to be determined. Therefore, we first developed a mixture of acetone and toluene as dissolvent for efficient lifting off QDs on thin resist (see Supporting Information S2 and S3 for details). Relying on this optimal lift-off process, the threshold resist thickness allowing for lifting off a LB film of $\sim 10 \mathrm{~nm}$ diameter QDs without redeposition was determined to be around $\sim 20 \mathrm{~nm}$. To verify the quality of the QD film prepared using this process, first we patterned $\mathrm{a} \sim 23 \mathrm{~nm}$ thick resist layer deposited on a silicon substrate with microscale feature size and then carried out the lift-off process. Figure $1 \mathrm{~b}$ shows the result, indicating that the LB technique allows transferring a uniform monolayer of QDs onto the patterned substrate and that the lift-off process works efficiently without leaving any unwanted residual QDs or resist. The excellent performance of the lift-off process can clearly be seen in Figure 1c, where the enlarged SEM image of the corner of the pattern distinctly shows a sharp boundary between the monolayer of QDs and the lifted area, without peeling off the patterned QDs. Figure 1d presents the morphology of the QD film at higher magnification, showing a nearly close-packed film except for a few tiny voids within the film, possibly caused by the size dispersion of the QDs or small aggregations in the QD solution. In Figure 1d, it can also be learned that the QD film can be well preserved after our lift-off process, thanks to the sufficient adhesion of QDs to the substrate.

Using the optimized lift-off process and a resist thickness near the previously determined threshold value, we also achieved nanoscale patterning of QD films. In Figure 2, we show patterning results with various shapes and different feature sizes. First, we patterned the QDs in lines and crossings with various widths, ranging from 30 to $250 \mathrm{~nm}$, as shown in Figure 2a,b. The lift-off process is demonstrated to be efficient for nanoscale patterns down to $30 \mathrm{~nm}$ feature size without any observable redeposition of free QDs or tearing of patterns. Moreover, it can be seen that the LB deposition is capable of transferring the QD film to the substrate through a $30 \mathrm{~nm}$ trench pattern in a $20 \mathrm{~nm}$ thick resist film, although as the magnified view for the $30 \mathrm{~nm}$ design in Figure $2 \mathrm{~b}$ shows there is an evident loss of QDs because of the non-negligible sidewall effect in the narrowest trenches. In fact, it appears that the breaking up of the LB film by the resist sidewalls randomizes the QD deposition at the pattern edges. This probably reflects the dominance of QD-substrate interactions over QD-QD interparticle interactions. ${ }^{35}$ As can be seen at the top of the 31 $\mathrm{nm}$ strip shown in Figure $2 \mathrm{~b}$, this can favor the deposition of QDs as individual entities on the resist sidewalls or the substrate over the systematic assembly of QDs in $2 \mathrm{D}$ or even $3 \mathrm{D}$ aggregates.

In patterns of $60 \mathrm{~nm}$ and wider on the other hand, a nearly perfect closed-packed film is formed, implying that the quantity of QDs can be actually determined, very important for the quantitative study of QD properties and their interaction with the environment. To verify preservation of the PL in the QDs, we carried out micro-PL measurements on the patterned QDs. The result shown in the inset of Figure 2a clearly indicates that the emission properties of the QDs are well preserved after LB deposition and the lift-off process, verifying further study is indeed of practical value. Similarly, we successfully patterned QDs in microring shapes with different widths (see Figure $2 \mathrm{c}, \mathrm{d})$. Finally, using a mask consisting of holes with different diameters we found uniformly filled QD patches can be formed 
for holes of $60 \mathrm{~nm}$ diameter, as shown in Figure 2e. Compared to the line and ring patterns these hole patterns are more difficult to form. To fill the resist hole the LB film now has to bend in all directions. When the size of the hole becomes comparable to the thickness of the resist, given the increasing ratio of the sidewall to hole area, the proportion of QDs deposited on the resist sidewalls that are eventually lifted off significantly increases while the probability for the QDs to remain in the target hole site on the substrate decreases. As shown in the Supporting Information, a reduction of the diameter of the hole in the resist also induces a more random deposition of the QDs on the substrate, similar to the edge effect observed with strip patterns. In the case of $47 \mathrm{~nm}$ wide holes, for example (see Figure S4, Supporting Information), this leads to deposits containing only a few, often unconnected QDs. This suggests that a further reduction of the resist hole diameter to dimensions comparable to the size of the QD itself, may lead to the trapping of a single $\mathrm{QD}$ in the resist hole.

To assess upfront the probability of trapping individual QDs on predefined spots of a substrate using a resist pattern consisting of an array of holes with small diameter and a certain resist thickness (see Figure 3a,b), we build on the conclusion (a)

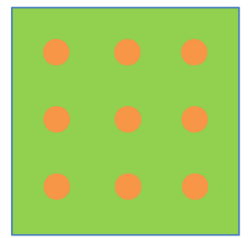

(c)

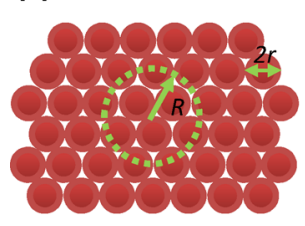

(b)
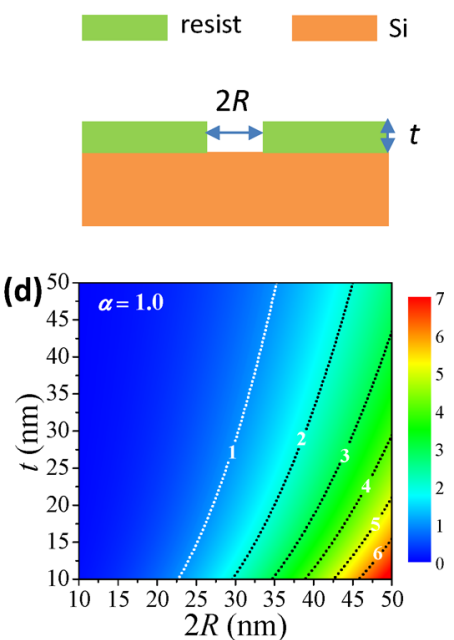

Figure 3. (a) Schematics of the array of hole patterns, (b) the side view of the hole with a diameter of $2 R$ and a resist thickness of $t$, and (c) the ideal close-packed QD film with the green-dot circle designated as the opening of the resist hole under the LB film. (d) Contour image of the calculated $N$ as a function of the resist thickness $t$ and hole diameter $2 R$ for QDs with an effective radius of $r=6.5 \mathrm{~nm}$ and $\alpha=1.0$.

that the resist sidewalls break apart the LB film resulting in a randomized deposition of QDs on the resist sidewalls and the substrate. The probability of retaining a given number of QDs on the substrate will then depend on the $\mathrm{QD}$ diameter and the surface areas of the hole and the resist sidewall. More specifically, the QD LB film can be described as a hexagonal close-packed (hcp) plane consisting of spheres with constant diameter (2r) as shown in Figure 3c. Assuming a resist thickness $t$ and hole radius $R$, the hole area $S_{\mathrm{h}}$ on the substrate and the sidewall area $S_{\mathrm{s}}$ of resist are simply calculated as $S_{\mathrm{h}}=$ $\pi R^{2}$, and $S_{\mathrm{s}}=2 \pi R t$, respectively. On the other hand, the number of QDs $n$ overlapping with the hole area can approximately be expressed as

$$
n=\Gamma \frac{S_{\mathrm{h}}}{\pi r^{2}}
$$

Here, $\Gamma$ is the packing fraction for the two-dimensional hcp lattice given by $\Gamma=\pi / 2 \sqrt{3}$. On the basis of our previous discussion, we neglect interparticle interactions and postulate that these $n$ QDs are randomly deposited over the substrate area $S_{\mathrm{h}}$ and a fraction $\alpha$ of the sidewall area $S_{\mathrm{s}}$ and that only the QDs actually deposited on the substrate will be retained after the lift-off process. The average number of QDs $N$ retrieved on the substrate is then given by the product of $n$ and the ratio of the accessible substrate area and the total area, substrate and sidewalls, available to the $n$ QDs

$$
N(r, R, t, \alpha)=\Gamma \frac{S_{\mathrm{h}}}{\pi r^{2}} \frac{S_{\mathrm{h}}}{S_{\mathrm{h}}+\alpha S_{\mathrm{s}}}
$$

To test this model, we analyze the number of QDs deposited on a line formed using a photoresist trench only 35-40 nm across. As shown in Figure 2b, the number of deposited QDs can be readily obtained by counting whereas it can be predicted using eq 2. Considering the uncertainty on the width of the resist trench, we find that the actual number of deposited QDs matches the prediction for $\alpha$ ranging from 0.7 to 1.0 with a best match obtained for $\alpha \approx 0.85$ (see Supporting Information S5).

We therefore use the above model to calculate the distribution of $N$ from eq 2 as a function of $R$ and $t$ for a given $r$ and $\alpha$. In Figure 3d, we show the calculated map of $N$ for the QDs used in our experiment with $r$ of $6.5 \mathrm{~nm}$ obtained by measuring the average center-to-center distance between LB QDs under SEM and taking $\alpha=1.0$. To achieve maximal probability of single-QD patterning, intuitively we should adopt such parameters of $R$ and $t$ that the value of $N$ can be maintained around 1.0 as indicated by the white dot line in Figure $3 \mathrm{~d}$. The calculated map clearly shows that $N$ is very sensitive to the diameter of the patterned hole. For $N \sim 1.0$ and resist thickness ranging from 10 to $50 \mathrm{~nm}$, the allowed working diameter window is only $\sim 12 \mathrm{~nm}$. Increasing the hole size above $35 \mathrm{~nm}$ results in a larger tolerance on the exact diameter but also leads to a dramatic increase in the optimal resist thickness $(>50 \mathrm{~nm})$ and a corresponding increase in aspect ratio $t / 2 R$. Intuitively it will be more difficult to coat a LB film conformally into a high aspect ratio hole. Furthermore, the relative fraction of QDs attached to the sidewall versus those attached on the substrate will increase leading to a larger variation on the number of QDs left on the surface. To the contrary, for smaller diameter of the hole, the required thickness $t$ (e.g., $t \sim 10 \mathrm{~nm}$ at $2 R \sim 22 \mathrm{~nm}$ ) for obtaining $N$ $\sim 1.0$ can be significantly reduced, decreasing the aspect ratio and facilitating deposition of the QD film. As discussed above, however, given a particular QD size, there exists a threshold thickness $t$ for the resist to allow for a successful lift-off process without residual QDs. For our QDs, this threshold thickness is $\sim 20 \mathrm{~nm}$ with a corresponding optimal hole diameter of $27 \mathrm{~nm}$ for $N \sim 1.0$ as can be derived from Figure 3d. The minimum hole diameter we can stably define in our EBL system however is $30-32 \mathrm{~nm}$ with a corresponding optimal resist thickness of $30-36 \mathrm{~nm}$.

To achieve optimal single QD deposition, we therefore patterned a $5 \times 5$ array of holes with diameter $\sim 31 \mathrm{~nm}$ in a 33 $\mathrm{nm}$ thick resist layer. After LB deposition and lift-off we observed and counted the number of QDs at each site via highresolution SEM. In Figure 4a, we show the collection of SEM images of the $5 \times 5$ dot patterns. We have eight cases of single- 
(a)

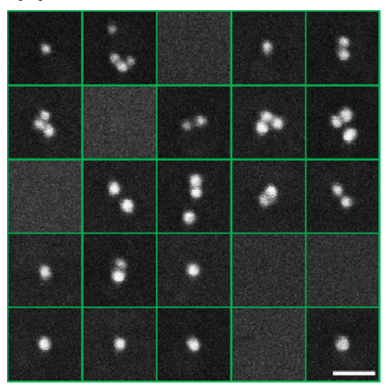

(b)

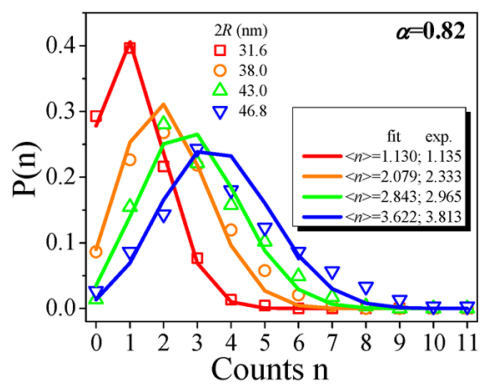

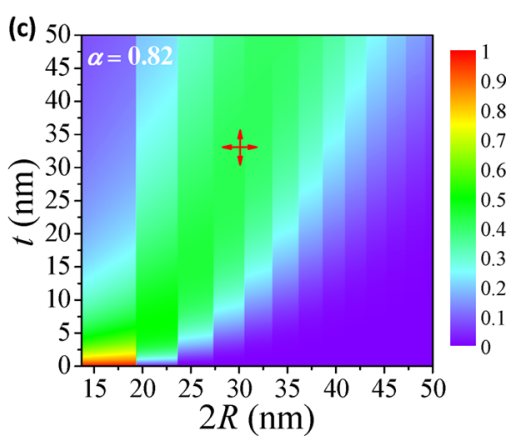

Figure 4. (a) SEM images of $5 \times 5$ dot patterns of QDs by using the resist hole of $31.6 \mathrm{~nm}$ diameter and $\sim 33 \mathrm{~nm}$ thickness. All images have the same scale bar of $50 \mathrm{~nm}$ as shown in the image at the right bottom corner. (b) The experimental (scatters) and binomial fitting (solid lines) distribution of the probability $P(n)$ as a function of the count of QDs for different diameters of the resist hole by counting 300 dot patterns, respectively. (c) The calculated probability of single-QD count $P(n=1)$ as a function of the diameter $2 R$ of hole and resist thickness $t$ by using a binomial distribution $B(n, p)$, with the red cross indicating the probability of $\sim 42 \%$ at $2 R=30 \mathrm{~nm}$ and $t=33 \mathrm{~nm}$.

QD occupancy for a total of 25 holes, equivalent with a singleQD yield of $32 \%$. The deviation of the number of QDs is pretty small and most holes end up with 0-3 QDs. In order to obtain a statistically more reliable distribution, we inspected patterns of 300 holes each for four different hole diameters. The results are shown in Figure $4 \mathrm{~b}$ using a different symbol for each hole diameter. For a diameter of $31.6 \mathrm{~nm}$ the probability of obtaining a single-QD reaches a peak value of $\sim 40 \%$ and the deviation of the distribution is only $\sim 2$ counts. When increasing the hole diameter, the single-QD probability decreases rapidly (only $\sim 8 \%$ for $46.8 \mathrm{~nm}$ diameter) and the peak of the probability shifts to larger $\mathrm{QD}$ counts. The distribution also broadens to about $\sim 3.5$ counts for a hole diameter of $46.8 \mathrm{~nm}$. The shift of the probability peak is intuitively understood from the $N$ map shown in Figure $3 \mathrm{~d}$ : when increasing the diameter for fixed resist thickness the expected number of QDs deposited on the substrate distinctively increases. Even so, the average number of deposited QDs $N$ as shown in Figure 3d only gives a partial description of the experimentally observed distribution curves, without accounting for, for example, their absolute position and magnitude or their broadening with increasing hole diameter. Moreover, it does not allow us to determine the upper limit for the probability of single-QD positioning in the experiment.

To assess the statistical characteristics behind the experimentally measured distribution for the probability of QD counts, we further build on the randomizing effect of the resist sidewall on the QD deposition. This allows us to look at the deposition of a QD on the substrate as an event that has a success probability $p$ given by the ratio between the accessible substrate area and the total area available, expressed as

$$
p=\frac{S_{\mathrm{h}}}{S_{\mathrm{h}}+\alpha S_{\mathrm{s}}}
$$

Here $S_{\mathrm{h}}, S_{s}$, and $\alpha$ were defined previously. Since $n$ QDs are deposited within each hole, the probability distribution of the number of QDs deposited on the substrate can then be approximated by a binomial distribution $B(n, p)$ with $n$ trials that each have a success probability $p$. In this way, by calculating $B(n, p)$ for a given $t, R$, and $\alpha$ we can calculate the probability distribution for the number of QDs deposited on the substrate.

First, we determine the a priori unknown parameter $\alpha$ by fitting the function $B(n, p)$ with $n(R, t)$ and $p(R, t, \alpha)$ to the experimentally obtained distribution curves for $t=33 \mathrm{~nm}$ and $2 R=(31.6,38.0,43.0,46.8 \mathrm{~nm})$. In this procedure, $n(R, t)$ as calculated using eq 1 was rounded to the closest lower integer value, that is, the maximum number of QDs that fit into the patterned hole, and $p(R, t, \alpha)$ was obtained from expression (3). Using a least-mean-square algorithm with $\alpha$ as the only free parameter we obtain $\alpha=0.82$ for an optimal fit, which is in close agreement to the value found previously for QD strip patterns. The result is shown in Figure $4 \mathrm{~b}$ in solid lines, showing excellent agreement with the experimental results, especially for the smallest diameter of $31.6 \mathrm{~nm}$. With increase of the diameter, the shift of the probability peak and the broadening of the distribution in the fits exactly represent the features of the experimental data. Note that we adopt an identical value for the parameter $\alpha$ for all fits and the good correspondence between experiment and fitting verifies our assumption that the sidewall area contributes to a certain proportion of the total deposition area in the case of small diameter hole patterns. The insert panel of Figure $4 \mathrm{~b}$ shows the mean $\langle n\rangle$ calculated for both the experimental and the fitted data. The discrepancy between both is less than $10 \%$ again indicating the suitability of the binomial distribution model for the description of the behavior of the deposition process of a LB QD film in a narrow hole pattern. Also, note that for the largest holes the binomial distribution underestimates the fraction of deposits containing more than five QDs. This may reflect the original observation that the LB film is only disturbed around the resist sidewalls, making that for larger resist holes the original LB film will be retrieved in the center of the deposit.

Although we experimentally achieved a single-QD pattering probability as high as $40 \%$, it is still interesting to explore the upper limit of this probability in the present technique. Relying on the above model, we therefore calculate the single-QD count probability $P(n=1)$ as a function of the diameter $2 R$ of the hole and the resist thickness $t$ with the experimentally obtained value $\alpha=0.82$. The result is shown in Figure $4 \mathrm{c}$. Note that the discontinuity at certain diameters stems from the discreteness of the integer $n$. Figure $4 \mathrm{c}$ shows that it is theoretically possible to accomplish a probability close to $100 \%$ for an infinitely thin resist thickness $(<5 \mathrm{~nm})$. However, we have already established that such a thin resist is not compatible with a successful and residue-free lift-off process. Taking into account the threshold thickness of $t \sim 20 \mathrm{~nm}$ for the resist layer, a probability of $\sim 45 \%$ theoretically can be reached. Considering the resolution limitation of our EBL system for the definition of the hole diameter $(30-32 \mathrm{~nm})$, the calculation 
shows a maximal probability of $\sim 42 \%$ for single-QD positioning as indicated by the red cross in Figure 4c, very close to our experimental result of $\sim 40 \%$. Using larger size quantum dots does not substantially increase this probability but does relax the requirements on the patterned holes, both in absolute size and in acceptable size variation.

In summary, using high quality LB deposition and a residuefree lift-off process, we experimentally demonstrated both nanoscale and single-dot patterning of colloidal QD-films. Feature sizes down to $\sim 30 \mathrm{~nm}$ for a continuously uniform film of QDs and a yield up to $40 \%$ for single-QD positioning are obtained. To describe the experimental processes a theoretical model was proposed. The good agreement between the experiment and the numerical model reveals that the deposition behavior of QDs onto a substrate and the experimental distribution of $\mathrm{QD}$ counts can be described by a binomial distribution, providing valuable guidance in the realization of single-QD patterns with an expected yield. The presently developed patterning technology for QDs provides an efficient tool both for the fundamental study of the properties of standalone QDs and for the quantitative investigation of the interaction between QDs and their environment such as onchip photonic or electronic devices. We believe that this technique will open up various novel applications relying on QDs and represents a practically significant step toward the development of real optoelectronic devices based on QDs, in particular those exploiting a single-QD emitter.

\section{ASSOCIATED CONTENT}

\section{S Supporting Information}

The Supporting Information is available free of charge on the ACS Publications website at DOI: 10.1021/acs.nanolett.5b03068.

The morphology of as-deposited QD film on patterned substrate, the detailed experiments for optimization of lift-off process, the investigation of the threshold thickness of resist for clean lift-off, and patterning results with an array of resist hole and line mask. (PDF)

\section{AUTHOR INFORMATION}

\section{Corresponding Author}

*E-mail: dries.vanthourhout@intec.ugent.be. Phone: +32-9-264 3438. Fax: +32-9-264 3593.

\section{Author Contributions}

The manuscript was written through contributions of all authors. All authors have given approval to the final version of the manuscript.

\section{Notes}

The authors declare no competing financial interest.

\section{ACKNOWLEDGMENTS}

The authors acknowledge the ERC-ULPPIC, H2020-MSCA phonsi and the IAP Photonics@be projects for financial support. The authors also acknowledge Dr. Zhechao Wang for help with e-beam lithography and Dr. Yolanda Justo for help with Langmuir-Blodgett technique.

\section{REFERENCES}

(1) Pattantyus-Abraham, A. G.; Qiao, H. J.; Shan, J.; Abel, K. A.; Wang, T. S.; van Veggel, F. C. J. M.; Young, J. F. Nano Lett. 2009, 9 (8), 2849-2854.
(2) Omari, A.; Geiregat, P.; Van Thourhout, D.; Hens, Z. Opt Express 2013, 21 (20), 23272-23285.

(3) Dang, C.; Lee, J.; Breen, C.; Steckel, J. S.; Coe-Sullivan, S.; Nurmikko, A. Nat. Nanotechnol. 2012, 7 (5), 335-339.

(4) Guilhabert, B.; Foucher, C.; Haughey, A. M.; Mutlugun, E.; Gao, Y.; Herrnsdorf, J.; Sun, H. D.; Demir, H. V.; Dawson, M. D.; Laurand, N. Opt. Express 2014, 22 (6), 7308-7319.

(5) Mashford, B. S.; Stevenson, M.; Popovic, Z.; Hamilton, C.; Zhou, Z. Q.; Breen, C.; Steckel, J.; Bulovic, V.; Bawendi, M.; Coe-Sullivan, S.; Kazlas, P. T. Nat. Photonics 2013, 7 (5), 407-412.

(6) Shirasaki, Y.; Supran, G. J.; Bawendi, M. G.; Bulovic, V. Nat. Photonics 2012, 7 (1), 13-23.

(7) Konstantatos, G.; Howard, I.; Fischer, A.; Hoogland, S.; Clifford, J.; Klem, E.; Levina, L.; Sargent, E. H. Nature 2006, 442 (7099), 180183.

(8) Zhang, C. Y.; Yeh, H. C.; Kuroki, M. T.; Wang, T. H. Nat. Mater. 2005, 4 (11), 826-831.

(9) Gupta, S.; Waks, E. Opt. Express 2013, 21 (24), 29612-29619.

(10) Qualtieri, A.; Pisanello, F.; Grande, M.; Stomeo, T.; Martiradonna, L.; Epifani, G.; Fiore, A.; Passaseo, A.; De Vittorio, M. Microelectron. Eng. 2010, 87 (5-8), 1435-1438.

(11) Pompa, P. P.; Martiradonna, L.; Della Torre, A.; Della Sala, F.; Manna, L.; De Vittorio, M.; Calabi, F.; Cingolani, R.; Rinaldi, R. Nat. Nanotechnol. 2006, 1 (2), 126-130.

(12) Michler, P.; Imamoglu, A.; Mason, M. D.; Carson, P. J.; Strouse, G. F.; Buratto, S. K. Nature 2000, 406 (6799), 968-970.

(13) Wang, X. Y.; Ren, X. F.; Kahen, K.; Hahn, M. A.; Rajeswaran, M.; Maccagnano-Zacher, S.; Silcox, J.; Cragg, G. E.; Efros, A. L.; Krauss, T. D. Nature 2009, 459 (7247), 686-689.

(14) Osovsky, R.; Cheskis, D.; Kloper, V.; Sashchiuk, A.; Kroner, M.; Lifshitz, E. Phys. Rev. Lett. 2009, 102 (19), 197401-4.

(15) Beyler, A. P.; Marshall, L. F.; Cui, J.; Brokmann, X.; Bawendi, M. G. Phys. Rev. Lett. 2013, 111 (17), 177401-5.

(16) Li, L.; Tian, G. J.; Luo, Y.; Brismar, H.; Fu, Y. J. Phys. Chem. C 2013, 117 (9), 4844-4851.

(17) Park, Y. S.; Bae, W. K.; Padilha, L. A.; Pietryga, J. M.; Klimov, V. I. Nano Lett. 2014, 14 (2), 396-402.

(18) Grinbom, G. A.; Saraf, M.; Saguy, C.; Bartnik, A. C.; Wise, F.; Lifshitz, E. Phys. Rev. B: Condens. Matter Mater. Phys. 2010, 81 (24), 245301-7.

(19) Diaconescu, B.; Padilha, L. A.; Nagpal, P.; Swartzentruber, B. S.; Klimov, V. I. Phys. Rev. Lett. 2013, 110 (12), 127406-5.

(20) Maturova, K.; Nanayakkara, S. U.; Luther, J. M.; van de Lagemaat, J. Nano Lett. 2013, 13 (6), 2338-2345.

(21) Qualtieri, A.; Morello, G.; Spinicelli, P.; Todaro, M. T.; Stomeo, T.; Martiradonna, L.; De Giorgi, M.; Quelin, X.; Buil, S.; Bramati, A.; Hermier, J. P.; Cingolani, R.; De Vittorio, M. New J. Phys. 2009, 11, 033025.

(22) Yuan, C. T.; Wang, Y. C.; Cheng, H. W.; Wang, H. S.; Kuo, M. Y.; Shih, M. H.; Tang, J. J. Phys. Chem. C 2013, 117 (24), $12762-$ 12768.

(23) Farahani, J. N.; Pohl, D. W.; Eisler, H. J.; Hecht, B. Phys. Rev. Lett. 2005, 95 (1), 017402-4.

(24) Curto, A. G.; Volpe, G.; Taminiau, T. H.; Kreuzer, M. P.; Quidant, R.; van Hulst, N. F. Science 2010, 329 (5994), 930-933.

(25) Curto, A. G.; Taminiau, T. H.; Volpe, G.; Kreuzer, M. P.; Quidant, R.; van Hulst, N. F. Nat. Commun. 2013, 4, 1750.

(26) Hancu, I. M.; Curto, A. G.; Castro-Lopez, M.; Kuttge, M.; van Hulst, N. F. Nano Lett. 2014, 14 (1), 166-171.

(27) Lambert, K.; Moreels, I.; Van Thourhout, D.; Hens, Z. Langmuir 2008, 24 (11), 5961-5966.

(28) Werts, M. H. V.; Lambert, M.; Bourgoin, J. P.; Brust, M. Nano Lett. 2002, 2 (1), 43-47.

(29) Park, Y.; Roh, Y. G.; Kim, U. J.; Chung, D. Y.; Suh, H.; Kim, J.; Cheon, S.; Lee, J.; Kim, T. H.; Cho, K. S.; Lee, C. W. Nanotechnology 2012, 23 (35), 355302.

(30) Mentzel, T. S.; Wanger, D. D.; Ray, N.; Walker, B. J.; Strasfeld, D.; Bawendi, M. G.; Kastner, M. A. Nano Lett. 2012, 12 (8), 44044408. 
(31) Manfrinato, V. R.; Wanger, D. D.; Strasfeld, D. B.; Han, H. S.; Marsili, F.; Arrieta, J. P.; Mentzel, T. S.; Bawendi, M. G.; Berggren, K. K. Nanotechnology 2013, 24 (12), 125302.

(32) Zhang, Q.; Dang, C.; Urabe, H.; Wang, J.; Sun, S. H.; Nurmikko, A. Opt. Express 2008, 16 (24), 19592-19599.

(33) Jiang, M. M.; Kurvits, J. A.; Lu, Y.; Nurmikko, A. V.; Zia, R. Nano Lett. 2015, 15 (8), 5010-5016.

(34) Cirillo, M.; Aubert, T.; Gomes, R.; Van Deun, R.; Emplit, P.; Biermann, A.; Lange, H.; Thomsen, C.; Brainis, E.; Hens, Z. Chem. Mater. 2014, 26 (2), 1154-1160.

(35) Lambert, K.; Justo, Y.; Kamal, J. S.; Hens, Z. Angew. Chem., Int. Ed. 2011, 50 (50), 12058-12061. 